\title{
NOVEL METHOD FOR THE SYNTHESIS
}

\section{OF 4-(AZOL-5-YL)ISOXAZOLES}

\author{
V. A. Bakulev ${ }^{1}$, I. V. Efimov ${ }^{1}$, N. A. Belyaev ${ }^{1}$, S. S. Zhidovinov ${ }^{1}$, \\ Yu. A. Rozin ${ }^{1}$, N. N. Volkova ${ }^{1}$, A. A. Khabarova ${ }^{1}$, and O. S. El'tsov ${ }^{1}$
}

Keywords: isothiazoles, isoxazoles, nitrile oxides, oxadiazoles, 1,2,3-thiadiazoles, cycloaddition.

In contrast to monocyclic and condensed isoxazoles, which are widely represented in the scientific and patent literature, bicyclic assemblies of isoxazoles with other azoles are poorly studied $[1,2]$. In particular, isoxazoles linked to 1,2,3-thiadiazole and isothiazole rings have not been reported in the literature. Current methods for the preparation of 4-(azol-5-yl)isoxazoles are not numerous and include the construction of either an azole or an isoxazole ring. The formation of an isoxazole ring through reactions of various derivatives of 1,3-dicarbonyl compounds, $\beta$-ketoesters, $\beta$-ketonitriles, and malononitrile derivatives with hydroxylamine has been used for the preparation of 4-(azol-5-yl)isoxazoles [1]. The cycloaddition reaction of azolylacetylenes to nitrile oxides $[1,3]$ represents an alternative way of preparing this type of compound. Despite the good yields, for the majority of reactions these methods are limited by the poor availability of the starting compounds. The Suzuki cross-coupling reaction is complex in experimental settings and hence has a limited use for the synthesis of 4-(azol-5-yl)isoxazoles [4].

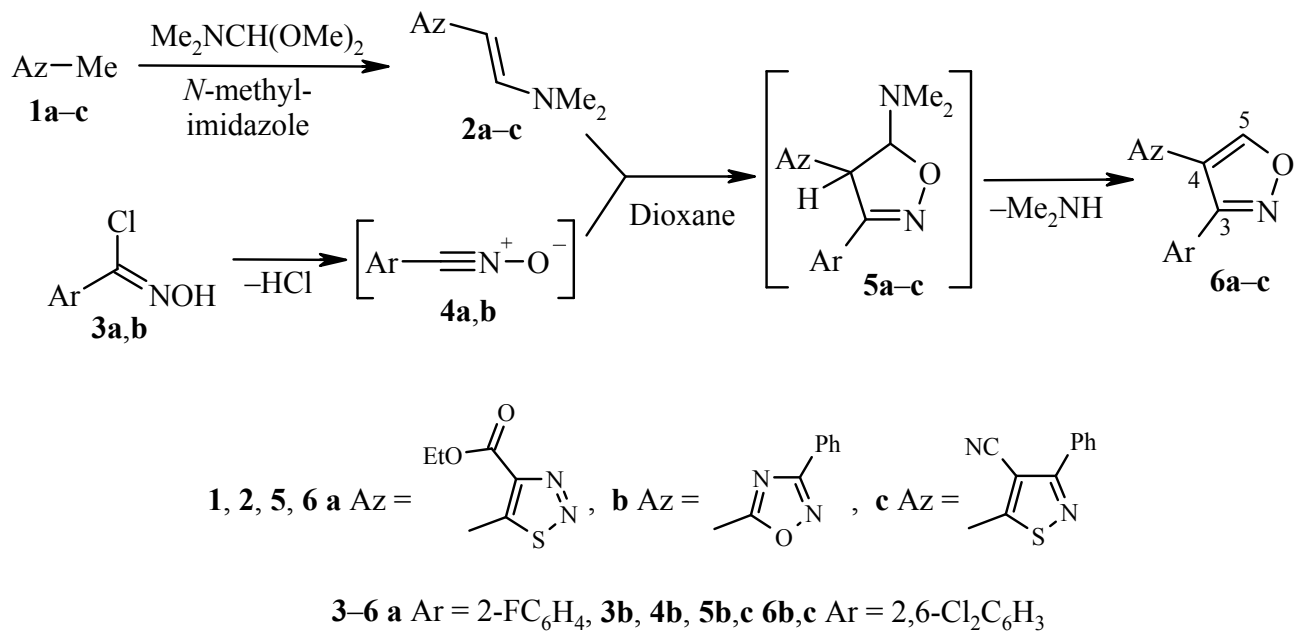

\footnotetext{
*To whom correspondence should be addressed, e-mail: vasiliy.bakulev@toslab.com.

${ }^{1}$ Ural Federal University named after the First President of Russia B. N. Yeltsin, 19 Mira St., Yekaterinburg 620002, Russia.
}

Translated from Khimiya Geterotsiklicheskikh Soedinenii, No. 12, pp. 2002-2005, December, 2012. Original article submitted September 27, 2012. 
Through systematic studies of the reactions of 3-azolylenamines [5, 6] we have developed a novel, experimentally simple, and regioselective method for preparing 4-(azol-5-yl)isoxazoles by the reaction of hydroxamoyl chlorides with 3-azolylenamines. In the case of the derivatives of 1,2,3-thiadiazole, 1,2,4-oxadiazole, and isothiazole 1a-c it was found that enamines 2a-c take part in a reaction with the hydroxamoyl chlorides 3a,b in the absence of bases, to give the bicyclic compounds 6a-c in $60-67 \%$ yields.

The mechanism of the reaction can be represented as a [3+2] cycloaddition of enamines $\mathbf{2 a - c}$ to the nitrile oxides $\mathbf{4 a}, \mathbf{b}$, which are formed in situ from the hydroxamoyl chlorides $\mathbf{3 a}, \mathbf{b}$ through elimination of $\mathrm{HCl}$. In the final stage, aromatization of the intermediate isoxazoline 5a-c occurs with the elimination of dimethylamine. The proposed mechanism can explain the regioselectivity of the enamine 2a-c reaction with hydroxamoyl chlorides $\mathbf{3 a}, \mathbf{b}$ as a result of the most favorable coulomb interaction of atom 3 of the enamine bearing the negative charge with the positively charged atom at position 3 of the nitrile oxides $\mathbf{4 a}, \mathbf{b}$.

It should be noted that the reactions of the 3-azolylenamines with hydroxamoyl chlorides and nitrile oxides have not been reported in the literature.

The structure of the compounds obtained was confirmed from ${ }^{1} \mathrm{H}$ and ${ }^{13} \mathrm{C}$ NMR, IR and UV spectroscopy, and from elemental analysis data. The signals for the dimethylamino group of compounds $\mathbf{2 b}, \mathbf{c}$ (present in the ${ }^{1} \mathrm{H}$ NMR spectra) were not observed in the ${ }^{13} \mathrm{C}$ NMR spectra due to their low intensity. We have previously observed a similar effect in the spectra of azolylenamines $[5,6]$.

IR spectra were recorded on a Bruker Alpha spectrometer (ATR method, ZnSe). ${ }^{1} \mathrm{H}$ NMR and ${ }^{13} \mathrm{C}$ NMR spectra were recorded on a Bruker Avance II 400 spectrometer (400 and $100 \mathrm{MHz}$, respectively) using DMSO-d $\mathrm{d}_{6}$ with TMS as internal standard. UV spectra were recorded on a Perkin-Elmer Lambda 45 spectrometer using $\mathrm{CH}_{2} \mathrm{Cl}_{2}$. Elemental analysis for $\mathrm{C}, \mathrm{H}, \mathrm{N}$ was performed on a Perkin-Elmer $2400 \mathrm{II}$ automatic analyzer and for sulfur by titration of sulfate anion after combustion in oxygen. Melting points were recorded on a Stuart SMP 3 apparatus.

Preparation of Enamines 2a-c (General Method). Dimethyl formamide dimethyl acetal $(265 \mu$, $2 \mathrm{mmol})$ and $N$-methylimidazole $(160 \mu \mathrm{l}, 2 \mathrm{mmol})$ were added to compound 1a-c $(1 \mathrm{mmol})$ and heated for $1 \mathrm{~h}$ at $80^{\circ} \mathrm{C}$. The reaction mixture was concentrated in vacuo, and hexane was added to the residue. The precipitate that formed was filtered, dried, and recrystallized from EtOH [7].

Ethyl 5-[(E)-2-(dimethylamino)ethenyl]-1,2,3-thiadiazole-4-carboxylate (2a). Yield $0.160 \mathrm{~g}(71 \%)$. Yellow crystals. Mp $114-115^{\circ} \mathrm{C}$. The melting point and ${ }^{1} \mathrm{H}$ NMR spectrum agreed with data given in the literature [6].

Dimethyl-(E)-2-(3-phenyl-1,2,4-oxadiazol-5-yl)ethenylamine (2b). Yield $0.150 \mathrm{~g}$ (70\%). Colorless crystals. Mp $79-83{ }^{\circ} \mathrm{C} .{ }^{1} \mathrm{H}$ NMR spectrum, $\delta$, ppm $(J, \mathrm{~Hz}): 3.02\left(6 \mathrm{H}\right.$, br. s, $\left.\mathrm{N}\left(\mathrm{CH}_{3}\right)_{2}\right) ; 5.02(1 \mathrm{H}, \mathrm{d}, J=13.1$, $\mathrm{N}-\mathrm{CH}=\mathrm{CH}) ; 7.38-7.50(3 \mathrm{H}, \mathrm{m}, \mathrm{H} \mathrm{Ph}) ; 7.67(1 \mathrm{H}, \mathrm{d}, J=13.1, \mathrm{~N}-\mathrm{CH}=\mathrm{CH}) ; 7.91-8.01(2 \mathrm{H}, \mathrm{m}, \mathrm{H} \mathrm{Ph}) .{ }^{13} \mathrm{C} \mathrm{NMR}$ spectrum, $\delta$, ppm: $76.4(\mathrm{CH}) ; 126.8,127.5,128.9,130.7(\mathrm{C} \mathrm{Ph}) ; 151.2(\mathrm{CH}) ; 166.8(\mathrm{C}-3) ; 178.0(\mathrm{C}-5)$. Found, \%: C 66.92; H 6.19; N 19.57. $\mathrm{C}_{12} \mathrm{H}_{13} \mathrm{~N}_{3} \mathrm{O}$. Calculated, \%: C 66.96; H 6.09; N 19.52.

5-[(E)-2-(Dimethylamino)ethenyl]-3-phenylisothiazole-4-carbonitrile (2c). Yield $0.176 \mathrm{~g} \quad(69 \%)$.

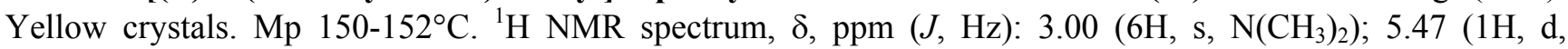
$J=13.1, \mathrm{~N}-\mathrm{CH}=\mathrm{CH}) ; 7.19(1 \mathrm{H}, \mathrm{d}, J=13.1, \mathrm{~N}-\mathrm{CH}=\mathrm{CH}) ; 7.38-7.53(3 \mathrm{H}, \mathrm{m}, \mathrm{H} \mathrm{Ph}) ; 7.98(2 \mathrm{H}, \mathrm{dd}, J=7.3$, $J=2.0, \mathrm{H} \mathrm{Ph}) .{ }^{13} \mathrm{C}$ NMR spectrum, $\delta$, ppm: $85.9(\mathrm{CH}) ; 97.2(\mathrm{C}-4) ; 116.3(\mathrm{CN}) ; 127.5,128.6,129.8,133.5$ $(\mathrm{C} \mathrm{Ph}) ; 147.1(\mathrm{CH}) ; 167.0(\mathrm{C}-5) ; 177.3$ (C-3). Found, \%: C 65.89; H 5.11; N 16.49; S 12.51. $\mathrm{C}_{14} \mathrm{H}_{13} \mathrm{~N}_{3} \mathrm{~S}$. Calculated, \%: C 65.85; H 5.13; N 16.46; S 12.56.

Ethyl 5-[3-(2-Fluorophenyl)isoxazol-4-yl]-1,2,3-thiadiazole-4-carboxylate (6a). 2-Fluorophenylhydroxamoyl chloride (3a) $(0.173 \mathrm{~g}, 1 \mathrm{mmol})$ was added to compound 2a $(0.227 \mathrm{~g}, 1 \mathrm{mmol})$, followed by absolute dioxane $(5 \mathrm{ml})$, and the mixture was stirred for $12 \mathrm{~h}$. The reaction mixture was concentrated in vacuo, hexane was added, and the precipitate was filtered off, dried, and recrystallized from EtOH. Yield $0.190 \mathrm{~g}(60 \%)$. White powder. Mp 110-111 ${ }^{\circ} \mathrm{C}$. IR spectrum, $v, \mathrm{~cm}^{-1}: 1721(\mathrm{C}=\mathrm{O})$. UV spectrum, $\lambda_{\max }, \mathrm{nm}(\log \varepsilon): 272(2.26)$. ${ }^{1} \mathrm{H}$ NMR spectrum, $\delta$, ppm $(J, \mathrm{~Hz}): 1.16\left(3 \mathrm{H}, \mathrm{t}, J=7.1, \mathrm{OCH}_{2} \mathrm{CH}_{3}\right) ; 4.16\left(2 \mathrm{H}, \mathrm{q}, J=7.1, \mathrm{OC}_{2} \mathrm{CH}_{3}\right) ; 7.26-7.40(2 \mathrm{H}$, m, H Ar); 7.50-7.66 (2H, m, H Ar); $9.64(1 \mathrm{H}, \mathrm{s}, \mathrm{H}-5) .{ }^{13} \mathrm{C}$ NMR spectrum, $\delta$, ppm: $13.6\left(\mathrm{OCH}_{2} \mathrm{CH}_{3}\right) ; 61.7$ 
$\left(\mathrm{OCH}_{2} \mathrm{CH}_{3}\right) ; 106.8(\mathrm{C}-4) ; 114.6\left(\mathrm{~d}, J_{\mathrm{C}-\mathrm{F}}=14.7, i-\mathrm{C} \mathrm{Ar}\right) ; 116.3\left(\mathrm{~d}, J_{\mathrm{C}-\mathrm{F}}=20.5, \mathrm{C}-3 \mathrm{Ar}\right) ; 125.2\left(\mathrm{~d}, J_{\mathrm{C}-\mathrm{F}}=2.9, \mathrm{C} \mathrm{Ar}\right)$; $131.2\left(\mathrm{~d}, J_{\mathrm{C}-\mathrm{F}}=2.2, \mathrm{C}\right.$ Ar); $133.1\left(\mathrm{~d}, J_{\mathrm{C}-\mathrm{F}}=8.1, \mathrm{C}-6 \mathrm{Ar}\right) ; 148.2,149.6(\mathrm{C}-4,5$ thiadiazole $) ; 156.3(\mathrm{C}-3) ; 158.9(\mathrm{~d}$, $\left.J_{\mathrm{C}-\mathrm{F}}=249.4, \mathrm{C}-2 \mathrm{Ar}\right) ; 159.3(\mathrm{C}=\mathrm{O}) ; 161.2(\mathrm{C}-5)$. Found, \%: C 52.71; H 3.23; N 13.13; S 10.14. $\mathrm{C}_{14} \mathrm{H}_{10} \mathrm{FN}_{3} \mathrm{O}_{3} \mathrm{~S}$. Calculated, \%: C 52.66; H 3.16; N 13.16; S 10.04.

5-[3-(2,6-Dichlorophenyl)isoxazol-4-yl]-3-phenyl-1,2,4-oxadiazole (6b) was obtained similarly from the enamine $\mathbf{2 b}$ and 2,6-dichlorophenylhydroxamoyl chloride (3b). Yield $0.239 \mathrm{~g}(67 \%)$. White powder. Mp 147- $150^{\circ} \mathrm{C}$. UV spectrum, $\lambda_{\max }, \mathrm{nm}(\log \varepsilon): 243$ (2.30). ${ }^{1} \mathrm{H}$ NMR spectrum, $\delta$, ppm: 7.44-7.65 (3H, m, H Ar); 7.65-7.80 (3H, m, H Ar); 7.85-7.99 (2H, m, H Ar); 10.32 (1H, s, H-5). ${ }^{13} \mathrm{C}$ NMR spectrum, $\delta$, ppm: 107.5 (C-4); 125.9, 127.4, 129.0, 129.7, 132.4, 133.7, 135.1 (C Ar); 156.5 (C-5); 164.9 (C-3); 167.8 (C-2,6 Ar); 167.9; 168.5 (C-3,5 oxadiazole). Found, \%: C 57.21; $\mathrm{H}$ 2.52; $\mathrm{N}$ 11.83. $\mathrm{C}_{17} \mathrm{H}_{9} \mathrm{Cl}_{2} \mathrm{~N}_{3} \mathrm{O}_{2}$. Calculated, \%: C 57.01; $\mathrm{H}$ 2.53; N 11.73 .

5-[3-(2,6-Dichlorophenyl)isoxazol-4-yl]-3-phenylisothiazole-4-carbonitrile $\quad(6 \mathbf{c})$ was prepared similarly from the enamine $2 \mathbf{c}$ and 2,6-dichlorophenylhydroxamoyl chloride (3b). Yield $0.258 \mathrm{~g}(65 \%)$. White powder. Mp $164-165^{\circ} \mathrm{C}$. IR spectrum, $v, \mathrm{~cm}^{-1}: 2220(\mathrm{C} \equiv \mathrm{N})$. UV spectrum, $\lambda_{\max }, \mathrm{nm}(\log \varepsilon): 262(2.41) .{ }^{1} \mathrm{H}$ NMR spectrum, $\delta$, ppm $(J, \mathrm{~Hz})$ : 7.52-7.64 (3H, m, H Ar); 7.70-7.84 (3H, m, H Ar); 7.85-7.99 (2H, m, H Ar); 10.08 $(1 \mathrm{H}, \mathrm{s}, \mathrm{H}-5) .{ }^{13} \mathrm{C}$ NMR spectrum, $\delta$, ppm: $109.9(\mathrm{C}-4) ; 113.6(\mathrm{CN}) ; 124.4 ; 127.6 ; 128.9 ; 129.1 ; 130.8 ; 131.8$ (C-4 isothiazole); 134.2; 135.0; 156.1 (C-3); 159.7 (C-5); 162.0 (C-5 isothiazole); 167.3 (C-2,6 Ar); 167.4 (C-3 isothiazole). Found, \%: C 57.36; $\mathrm{H}$ 2.26; $\mathrm{N} 10.51 ; \mathrm{S}$ 8.09. $\mathrm{C}_{19} \mathrm{H}_{9} \mathrm{Cl}_{2} \mathrm{~N}_{3} \mathrm{OS}$. Calculated, \%: C 57.30; $\mathrm{H} 2.28$; N 10.55; S 8.05.

The authors thank the Russian Foundation for Basic Research (grant 11-03-00579-a) for financial support.

\section{REFERENCES}

1. D. Giomi, F. M. Cordero, and F. Machetti, in: A. R. Katritzky, C. W. Rees, and E. F. V. Scriven (editors), Comprehensive Heterocyclic Chemistry III, Vol. 4, Pergamon Press, Oxford (2008), p. 365.

2. F. Giuntini, M. A. F. Faustino, M. G. P. M. S. Neves, A. C. Tomé, A. M. S. Silva, and J. A. S. Cavaleiro, Tetrahedron, 61, 10454 (2005).

3. G. Himbert, D. Faul, and M. Bars, Z. Naturforsch., B: J. Chem. Sci., 46, 955 (1991).

4. J. Loerstedt, X. Wu, and L. Krueger, WO Pat. Appl. 2011/042475.

5. Y. Shafran, Y. Rozin, T. Beryozkina, S. Zhidovinov, O. Eltsov, J. Subbotina, J. Leban, R. Novikova, and V. Bakulev, Org. Biomol. Chem., 10, 5795 (2012).

6. V. A. Bakulev, I. V. Efimov, N. A. Belyaev, Yu. A. Rozin, N. N. Volkova, and O. S. El'tsov, Khim. Geterotsikl. Soedin., 1900 (2011). [Chem. Heterocycl. Compd., 47, 1593 (2011)].

7. A. Sarkar, S. R. Roy, D. Kumar, C. Madaan, S. Rudrawar, and A. K. Chakraborti, Org. Biomol. Chem., 10, 281 (2012). 